\title{
Flat Panel Detector Angiographic CT in the Management of Aneurysmal Rupture During Coil Embolization
}

T. Engelhorn

SUMMARY: Angiographic CT (ACT) produces CT-like images within minutes in the angiography suite. We present the cases of 2 patients with intraprocedural aneurysmal rupture in which ACT enabled the neuroradiologist to rapidly assess the extent of bleeding during endovascular treatment. Additionally, ACT revealed pronounced rebleeding within the time between conventional CT and onset of treatment. In the management of aneurysmal rupture during coil embolization, ACT may be a valuable adjunct.

O. Ganslanc

W. Kalender

A. Doerfler

$\mathbf{T}$ he International Subarachnoid Aneurysm Trial study ${ }^{1}$ showed intracranial aneurysms, especially in the anterior circulation, to be treated more effectively with endovascular coiling because of a significant, more beneficial clinical outcome compared with neurosurgical aneurysmal clipping. Despite technical improvements in guidewires, catheters, and coils, rupture of an aneurysm during endovascular treatment is one of the most feared complications of endovascular aneurysmal therapy. If this complication happens, postprocedural CT is the method of choice to reveal the dimension of bleeding. In this situation, a radiologic tool to produce CT-like images within the angiographic suite would be desirable.

Angiographic CT (ACT) uses rotational, C-arm-mounted, flat panel detector technology capable of high-spatial resolution volumetric imaging. Rotational acquisitions with such detectors provide an almost CT-like contrast resolution in the angiography suite within minutes. ${ }^{2,3}$ This technique may improve procedural safety of aneurysmal coiling, and it may enhance our understanding of aneurysmal rupture during endovascular treatment by immediate visualization of the extent of subarachnoid hemorrhage.

\section{Technique}

Of a series of 74 patients with subarachnoid hemorrhage from aneurysmal rupture within the last 18 months, we retrospectively identified 2 patients with rebleeding during coil embolization.

In addition to a conventional head CT examination on a 4-section CT scanner (Somatom Volume Zoom; Siemens, Forchheim, Germany), ACT was routinely performed in patients with aneurysmal rupture directly before coil embolization and in cases of rupture after loose coiling as soon as the contrast extravasation stopped.

We performed data acquisition using a biplane, flat panel detector angiography system (Axiom Artis dBA; Siemens Medical Solutions; Erlangen, Germany) and commercially available software (DynaCT; Siemens), whereas postprocessing was performed with a separate

Received October 23, 2007; accepted after revision December 7

From the Departments of Neuroradiology (T.E., T.S., G.R., M.D., A.D.) and Neurosurgery (O.G.) and Institute of Medical Physics (W.K.), Friedrich-Alexander University ErlangenNuremberg, Erlangen, Germany.

Please address correspondence to Tobias Engelhorn, MD, Friedrich-Alexander University Erlangen-Nuremberg, Department of Neuroradiology, Schwabachanlage 6, D-91052 Erlangen, Germany; e-mail: tobias.engelhorn@nrad.imed.uni-erlangen.de

DOl 10.3174/ajnr.A1119 workstation (Leonardo; Siemens). We performed ACT data acquisition using the following parameters: acquisition time, 20 seconds; $1240 \times 960$ projection matrix; projection on $30 \times 40-\mathrm{cm}$ flat panel size; $217^{\circ}$ rotation angle, 538 images in total, increment $0.4^{\circ}$ /image. The standard system dose was $0.36 \times 10^{-6} \mathrm{~Gy} /$ image, so radiation dose to patients was similar to that of a conventional head CT dose, up to $20 \mathrm{mGy}$. Differentiation of soft tissue densities was approximately 10 Hounsfield units. ${ }^{2,3}$

Acquisition of ACT volume data was performed without administration of contrast agent. Postprocessing consisted of computed correction of beam hardening, ring artifacts, and scattered radiation. Multiplanar reconstructed images (MPRs) in the axial orientation parallel to the skull base with a section thickness of $8 \mathrm{~mm}$ (identical to conventional CT) were obtained for visualization of subarachnoid hemorrhage. Obtainable optimum in visibility was achieved with window values displayed circa 250 for window width (W) and 70 for window level (C), also allowing good assessment of CSF diversion with regard to potential hydrocephalus. The whole procedure of ACT acquisition and postprocessing took approximately 5 minutes.

\section{Case Reports}

\section{Patient 1}

A 45-year-old woman presented with acute subarachnoid hemorrhage (SAH; Fig 1A), clinically classified as Hunt and Hess grade II, and was found to have an aneurysm of the anterior communicating artery (diameter $5 \times 5 \mathrm{~mm}$, Fig $1 E$ ). Endovascular therapy was performed right after ventriculostomy on day 1 after SAH. Preprocedural ACT revealed clear rebleeding pronounced in the anterior interhemispheric gap and in the left frontal lobe compared with CT after ventriculostomy (Fig $1 B$ ). In addition, rebleeding occurred during diagnostic angiography before onset of therapy (Fig $1 F$ ). With use of standard techniques, the aneurysm was coiled with a series of detachable coils. After introduction of the first 2 coils, contrast extravasation stopped and ACT revealed no significant space-occupying rebleeding after rupture (Fig 1C). Coil embolization was continued with 6 additional coils without any problems. Arteriographic studies after placement of each coil to document appropriate coil positioning did not demonstrate any additional extravasation of contrast agent after introduction of the first 2 coils. The final angiogram showed complete occlusion of the aneurysm (Figs $1 G,-H$ ). Results of 

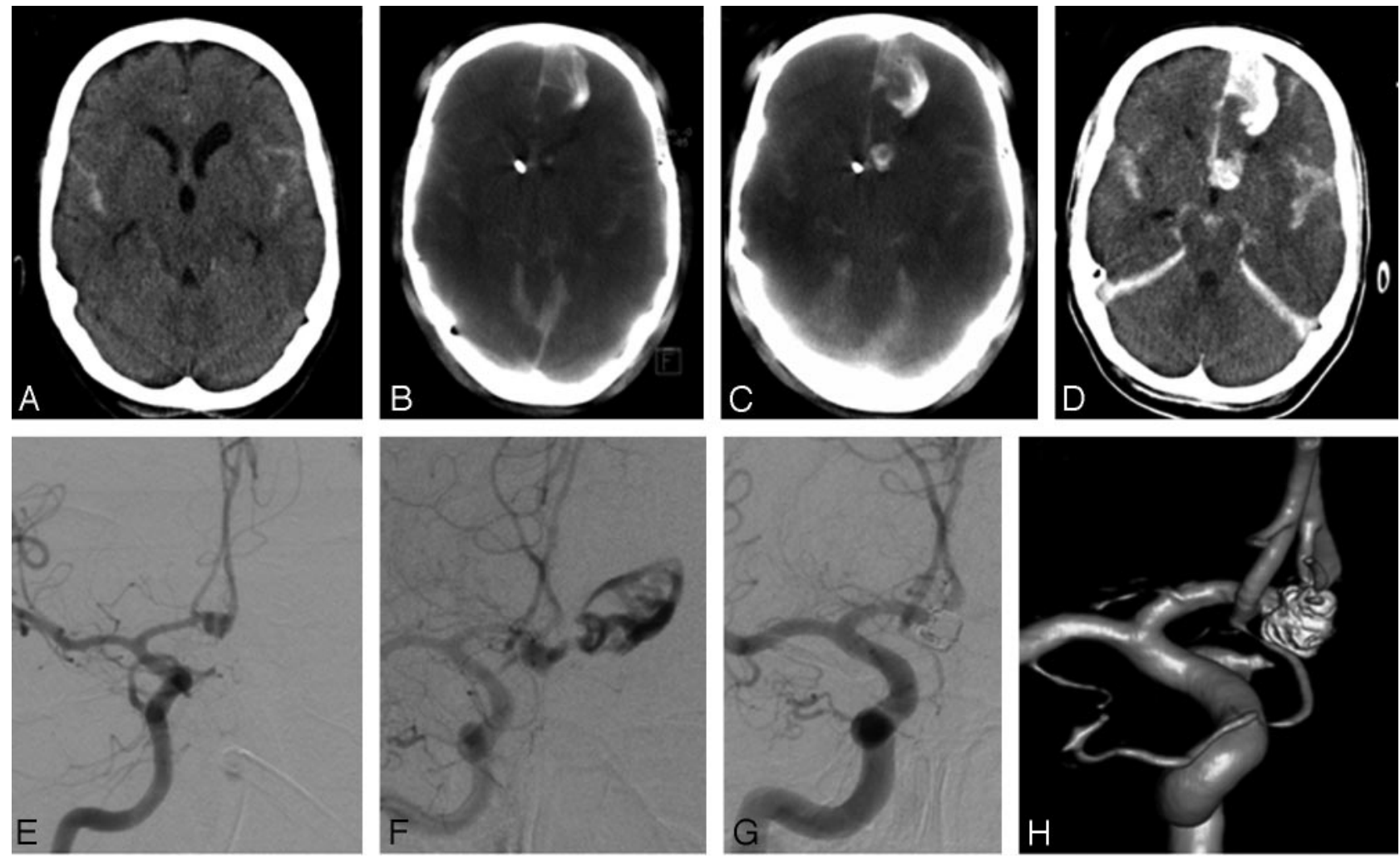

Fig 1. Patient 1. A, Initial axial CT scan before coil embolization shows only slight subarachnoid hemorrhage without intraparenchymal bleeding. $B$, Axial ACT scan reveals rebleeding mainly in the anterior interhemispheric gap and in the left frontal lobe before the onset of diagnostic angiography. $C$, Axial ACT scan after aneurysmal rupture and installation of the first 2 coils demonstrates no significant space-occupying rebleeding compared with initial ACT. D, Axial CT scan after coil embolization shows only subtle additional subarachnoid hemorrhage compared with intraprocedural ACT (C). E, Right internal carotid artery (ICA) arteriogram (anterior-posterior [AP] projection) reveals an anterior communicating artery aneurysm. F, Right ICA arteriogram (AP projection) demonstrates massive extravasation of contrast media before onset of endovascular therapy. $G$ and $H$, Right ICA arteriogram (AP projection) and rotational $3 \mathrm{D}$ angiography show complete embolization after installation of 8 Guglielmi detachable coils.

CT after the procedure showed only subtle rebleeding compared with intraprocedural ACT (Fig 1D).

\section{Patient 2}

A 43-year-old man presented with acute SAH (Fig 2A), clinically classified as Hunt and Hess grade II. Results on angiography revealed an irregular aneurysm of the basilar tip (diameter $4 \times 6 \mathrm{~mm}$, Fig $2 F$ ). Endovascular therapy was performed right after conventional CT (Fig 2A) on day 1 after SAH. Preprocedural ACT (Fig 2C) revealed no rebleeding compared with initial CT. After installation of the first coil, results of the arteriographic study revealed slight contrast extravasation (Figs 2G, $-H$ ), which stopped after installation of a second coil. Hereby, intraprocedural ACT (Fig 2D) revealed no significant increase in subarachnoid hemorrhage after aneurysmal rupture, and coil embolization was continued with 5 additional coils without any problems and without a hint of new rebleeding. The final angiogram results showed complete occlusion of the aneurysm (Fig 2J), and the conventional CT results after the procedure showed no rebleeding compared with intraprocedural ACT (Fig 2E).

The final outcome of both patients was good (good recovery ${ }^{1}$ according to the Glasgow Outcome Scale/GOS) and 6-month follow-up evaluation with angiography revealed no coil compaction or partial recanalization of the aneurysms.

\section{Discussion}

For patients undergoing embolization of an aneurysm, early recognition and management of complications such as rebleeding can significantly improve clinical outcomes. ${ }^{4}$ When a complication occurs during an endovascular procedure, the patient is usually transported emergently from the angiography suite to a CT scanner to determine a diagnosis. The new technology of a combined angiography/CT suite with flat panel detector technology for higher-resolution angiography is also capable of producing improved cone-beam volume CT images. Although images produced by ACT are not of the same quality as conventional CT, current ACT image quality is fully sufficient to assess the extent of intracranial hemorrhage when a complication is suspected. ${ }^{5}$

The cases of our 2 patients demonstrate that ACT can avoid transportation of the patient, resulting in less delay in critical decision making. Because ACT showed no significant increase in subarachnoid hemorrhage, none of the patients had to rapidly undergo neurosurgical intervention from space-occupying bleedings, and coil embolization could be finished without pressure of time, an important presumption for an attenuated packing result and reduced frequency of repeated treatment.

Additionally, ACT demonstrated rebleeding in patient 1 immediately before coil embolization (ie, this rebleeding occurred between conventional CT and transport to the angiog- 

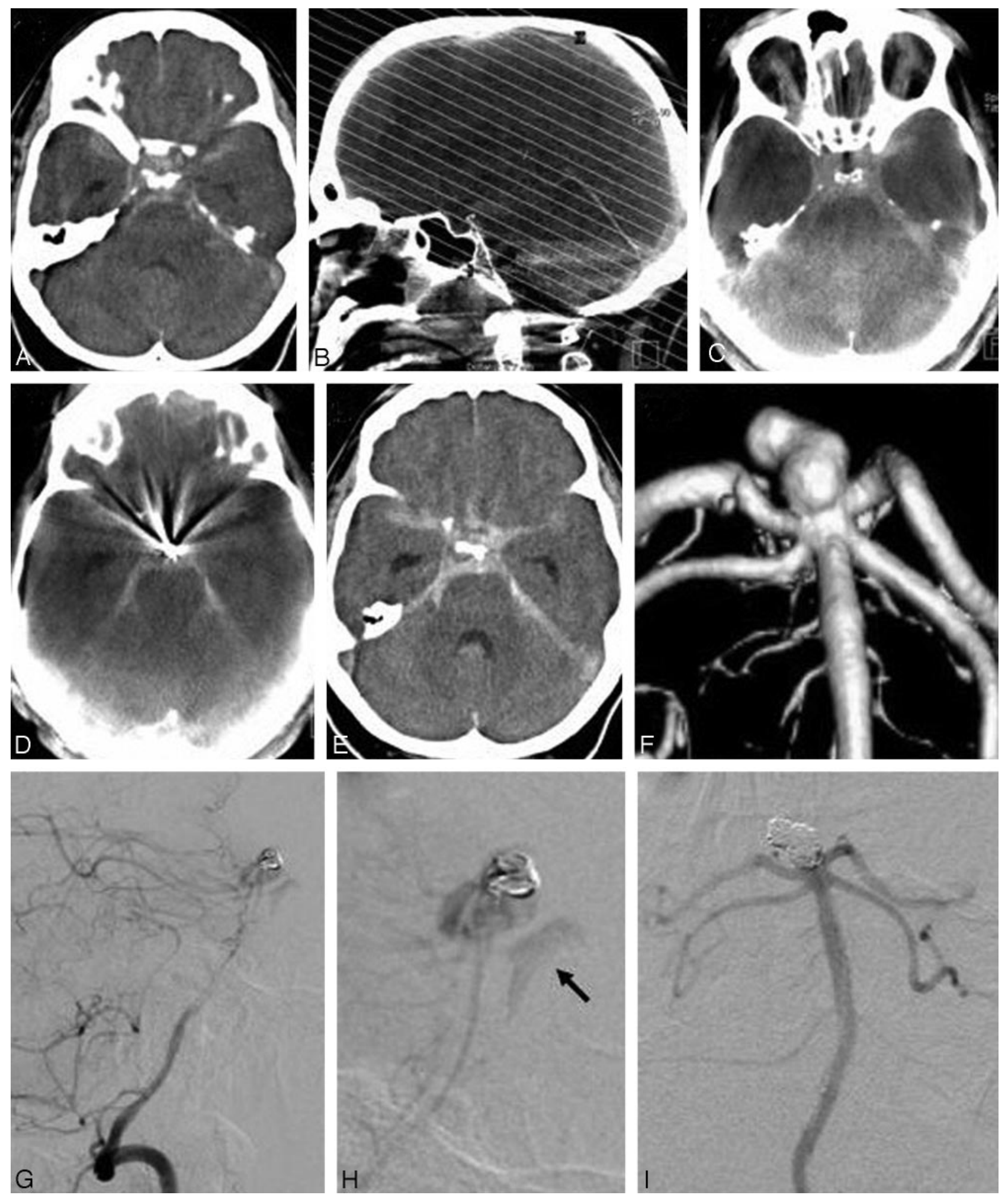

Fig 2. Patient 2. A, Axial CT scan before onset of endovascular treatment. $B$, After postprocessing, MPRs in axial orientation parallel to the skull base identical to conventional $C T$ were calculated. C, Axial ACT scan (preprocedural) reveals no rebleeding in the time between conventional CT and onset of angiography. $D$, Axial ACT scan (intraprocedural) after aneurysmal rupture and installation of 2 coils shows no significant increase in subarachnoid hemorrhage compared with preprocedural ACT. E, Axial CT scan after endovascular treatment shows no rebleeding after installation of the first 2 coils. F, Rotational 3D angiography shows an irregular, lobulated basilar tip aneurysm. $G$ and $H$, Basilar artery arteriogram (lateral projection) demonstrates slight extravasation of contrast media (arrow) after installation of the first coil. I, Basilar artery arteriogram (AP projection) shows complete embolization after installation of 7 coils.

raphy suite). Without ACT, this rebleeding would have been undetected until postprocedural CT, which suggests that this bleeding was related to endovascular treatment.

\section{Conclusion}

ACT enables detection of intracranial hemorrhage and its extent within the angiography suite, allowing earlier diagnosis 
and management of hemorrhagic complications such as rebleeding and treatment-derived aneurysmal rupture. In cases of rupture, the width of the ventricles and the dimension of subarachnoid hemorrhage can be assessed within minutes; hence, its use facilitates the management of rupture-related complications and improves safety and outcome of endovascular treatment.

\section{References}

1. Molyneux AJ, Kerr RS, Yu LM, et al. International subarachnoid aneurysm trial (ISAT) of neurosurgical clipping versus endovascular coiling in 2143 patients with ruptured intracranial aneurysms: a randomised comparison of effects on survival, dependency, seizures, rebleeding, subgroups, and aneurysm occlusion. Lancet 2005;366:809-17

2. Loose R, Wucherer M, Brunner T. Visualization of 3D low contrast objects by $\mathrm{CT}$ cone-beam reconstruction of a rotational angiography with a dynamic solid body detector. RoFo 2005;S1:PO160

3. Kalender WA. The use of flat-panel detectors for CT imaging. Radiologe 2003;43:379-87

4. Levy E, Koebbe CJ, Horowitz MB, et al. Rupture of intracranial aneurysms during endovascular coiling: management and outcomes. Neurosurgery 2001; 49:807-11; discussion 811-13

5. Heran NS, Song JK, Namba K, et al. The utility of DynaCT in neuroendovascular procedures. AJNR Am J Neuroradiol 2006;27:330-32 